\title{
BMJ Open Neural and clinical changes of cognitive behavioural therapy versus talking control in patients with major depression: a study protocol for a randomised clinical trial
}

Nariko Katayama (D) , ${ }^{1}$ Atsuo Nakagawa (D) , ${ }^{1,2}$ Chika Kurata, ${ }^{1}$ Yohei Sasaki, ${ }^{1}$ Dai Mitsuda, ${ }^{1}$ Shigetsugu Nakao, ${ }^{1}$ Sayuri Mizuno, ${ }^{1}$ Mire Ozawa, ${ }^{1}$ Yuko Nakagawa, ${ }^{1}$ Natsumi Ishikawa, ${ }^{1}$ Satoshi Umeda, ${ }^{3}$ Yuri Terasawa, ${ }^{3}$ Hajime Tabuchi, ${ }^{1}$ Toshiaki Kikuchi, ${ }^{1}$ Takayuki Abe, ${ }^{4}$ Masaru Mimura ${ }^{1}$

To cite: Katayama $\mathrm{N}$, Nakagawa A, Kurata C, et al. Neural and clinical changes of cognitive behavioural therapy versus talking control in patients with major depression: a study protocol for a randomised clinical trial. BMJ Open 2020;10:e029735. doi:10.1136/ bmjopen-2019-029735

- Prepublication history and additional material for this paper are available online. To view these files, please visit the journal online (http://dx.doi. org/10.1136/bmjopen-2019029735).

Received 08 February 2019 Revised 27 November 2019 Accepted 03 February 2020

Check for updates

(C) Author(s) (or their employer(s)) 2020. Re-use permitted under CC BY-NC. No commercial re-use. See rights and permissions. Published by BMJ.

For numbered affiliations see end of article.

Correspondence to

Dr Atsuo Nakagawa;

anakagawa@keio.jp

\section{ABSTRACT}

Introduction While major depression causes substantial distress and impairment for affected individuals and society, the effectiveness of cognitive behavioural therapy (CBT) in treating the condition has been established. However, the therapeutic mechanism underlying the efficacy of CBT remains unknown. This study aimed to describe a protocol for a randomised controlled trial that will measure the CBT-induced clinical and neural changes in patients with nonpsychotic major depression.

Methods and analysis The current study is a 16-week assessor-blinded, randomised, parallel-group trial with a 12-month follow-up as part of usual depression care at an outpatient clinic. Patients aged 20-69 years with major depressive disorder will be randomly assigned to receive either $\mathrm{CBT}$ in addition to their usual treatment or talking control in addition to their usual treatment for 16 weeks. The primary outcome is the functional changes in the brain areas that have been associated with futureoriented thinking at 16 weeks; secondary outcomes include changes in functional brain connectivity, severity and changes in the scores of objective and subjective clinical depression symptoms, proportion of responders and remitters and quality of life. The intention-to-treat analysis will be used.

Ethics and dissemination All protocols and the informed consent form are compliant with the Ethics Guideline for Clinical Research (Japanese Ministry of Health, Labour and Welfare). Ethical Review Committees at the Keio University School of Medicine have approved the study protocol (version 3, 11 September 2017). We will disseminate research findings to scientific and general audiences through national and international conference presentations as well as lay summaries to the general public, including mental health consumer and publications in international peer-reviewed psychiatry and brain imaging journals.

Trial registration number UMIN Clinical Trials Registry (UMIN000018155); Pre-results.
Strengths and limitations of this study

This study design is the first to attempt to elucidate the neurobiological underpinnings of future-oriented pessimistic thinking in patients with major depression following the receipt of cognitive behavioural therapy (CBT)

- This study is a prospective, randomised controlled study with blinded assessment.

- This study may provide neurobiological evidence for the efficacy of CBT in the treatment of major depression.

- The study's small sample size and single-site design may affect its generalisability.

\section{INTRODUCTION}

Major depressive disorder (MDD) is a highly prevalent psychiatric disorder that affects an approximate 300 million people globally. ${ }^{1}$ Although progress has been made in the treatment of this disorder, it has been estimated that more than one-third of patients with MDD do not respond satisfactorily to initial and subsequent antidepressant treatments, including combinations of pharmacotherapy and psychotherapy. ${ }^{2}$ Thus, there is an urgent need to develop novel and more effective therapeutic strategies.

It is has been established that cognitive behavioural therapy (CBT), the most published structured form of psychotherapy developed on the basis of Beck's cognitive theory, ${ }^{3}$ is effective in treating depression. ${ }^{4} 5$ Numerous randomised controlled studies have shown that CBT is superior to wait-list, non-specific controls and treatment as usual (TAU). ${ }^{6}$ Further evidence shows that combining psychotherapy and 
pharmacotherapy is more effective than pharmacotherapy alone. ${ }^{7}$ Our prior randomised controlled trial showed that patients with pharmacotherapy-resistant depression benefited from supplementing their medication management with $\mathrm{CBT}^{8}$

Patients with depression exhibit hyperactivity in the amygdala and hypoactivity in the prefrontal cortex ${ }^{9}$; it has been hypothesised that pharmacotherapy directly reduces the former (bottom-up effect), while CBT improves the latter and thus mitigates the excess activity of the amygdala (top-down effect). ${ }^{10}$ Over the past decade, several published neuroimaging studies have verified the therapeutic effect of CBT. The functional connectivity between brain regions is also associated with differential treatment outcomes for medications and CBT. ${ }^{11}$ However, the observed pattern of activation and connectivity in these areas varied among studies, and only one positronemission tomography study used reliable randomised controlled trials to obtain evidence. ${ }^{12} 13$

Patients with MDD tend to endorse pessimistic thinking due to the difficulty of imagining a positive future; this predisposes them to hopelessness. ${ }^{14}$ The Diagnostic and Statistical Manual of Mental Disorders 5th Edition and International Classification of Disease 10th Revision list pessimistic thinking about the future as one of the cardinal symptoms of MDD. Beck proposed that patients with MDD have specific irrational and pessimistic thoughts about future opportunities and prospects and that these negative cognitive biases cause symptoms of depression-not vice versa. ${ }^{15}$ This mechanism of symptom onset differentiates MDD from other psychiatric diseases, such as bipolar disorders or schizophrenia. In accordance with Beck's cognitive theory of depression, a recent systematic review of the empirical literature indicated that patients with MDD feature a less adaptive cognitive style, characterised by concrete and specific processing (ie, more maladaptive style characterised by abstract and overly general processing $)^{1617}$ and a reduced ability to imagine possible futures. ${ }^{18}$ In the context of cognitive behavioural therapy, improving the patient's ability to imagine possible futures is one of the most important processes, which includes planning and predicting events and outcomes and completing between-session assignments associated with prospective memory. ${ }^{19}$ Neuroimaging studies have shown that the medial frontopolar cortex, situated in the medial area of the anterior prefrontal cortex (Brodmann area (BA) 10), plays a key role in future-thinking in healthy individuals. ${ }^{20-22}$ It has been proposed that BA10 serves as an integrative centre for higher order emotional, cognitive and social processes. ${ }^{23}{ }^{24}$ In our previous study, we found that patients with MDD were associated with a greater propensity to engage in negative thinking about the distant future and exhibited increased activation in BA10 relative to healthy individuals when imagining the distant future. ${ }^{25}$ These obervations suggest that patients with MDD engage in pessimistic future thinking and experience difficulty in imagining their distant future, which is associated with abnormal patterns of frontal pole activity.
Dysfunction of the frontal pole during distant future thinking may reflect negative cognition concerning the future and may thus feature potential as a neuromarker for depression as well as indicate the need for interventions, such as CBT, that challenge negative dysfunctional cognition about the future.

Despite its clinical significance, no study has reported the neurobiological underpinnings of future-oriented pessimistic thinking in patients with MDD following the receipt of CBT. We therefore planned a phase II randomised controlled trial to examine the neural and clinical changes regarding future thinking in patients treated for MDD with CBT or talking control (TC). This paper aims to describe the study protocol of the current study.

\section{Study aims and hypothesis}

The primary aim of this study is to compare changes in brain activity underlying shifts in future thinking across 16 weeks of CBT and TC in patients with non-psychotic MDD. The secondary aim of the study is to investigate the effect of CBT on clinical and behavioural changes and shifts in neural functional connectivity relative to those of TC. The tertiary aim of the study is to assess therapy response by means of neurobiological measures.

We hypothesise that patients with MDD who receive CBT will show favourable cerebral haemodynamic changes in brain regions related to future thinking, such as diminished BA10 activity when imaging the distant future and achieve greater improvements in specific domains of cognition than those who receive TC.

\section{METHODS}

\section{Study design, setting and approval}

The current study is a single-site 16-week assessor-blinded, randomised controlled trial of two parallel groups with a 12-month follow-up period conducted as part of usual depression care at a university teaching hospital outpatient clinic (figure 1). Random treatment allocation will be done at the individual level. Patients will be recruited at the Keio University Hospital, a university teaching hospital located in central Tokyo, Japan.

\section{Patients}

Patients are eligible to be included in the study if they meet the following criteria: (1) outpatients with a diagnosis of MDD, as defined by the DSM 4th Edition (DSMIV) ${ }^{26}$ criteria for single or recurrent MDD without psychotic features, assessed by a trained psychiatrist with the Structured Clinical Interview for DSM-IV (SCID) ${ }^{27}$; (2) aged between 20 and 69 years; (3) experiencing at least moderate-level depression symptoms-at least 16 on the GRID-Hamilton Depression Rating Scale-17 item $\left(\text { GRID-HAMD }_{17}\right)^{28} 29$ and (4) competent and able to give informed consent.

Patients will be excluded from the study if they meet the following criteria: (1) have had past or current manic or 
Patients provided information on the study by treating psychiatrists and referred for eligibility

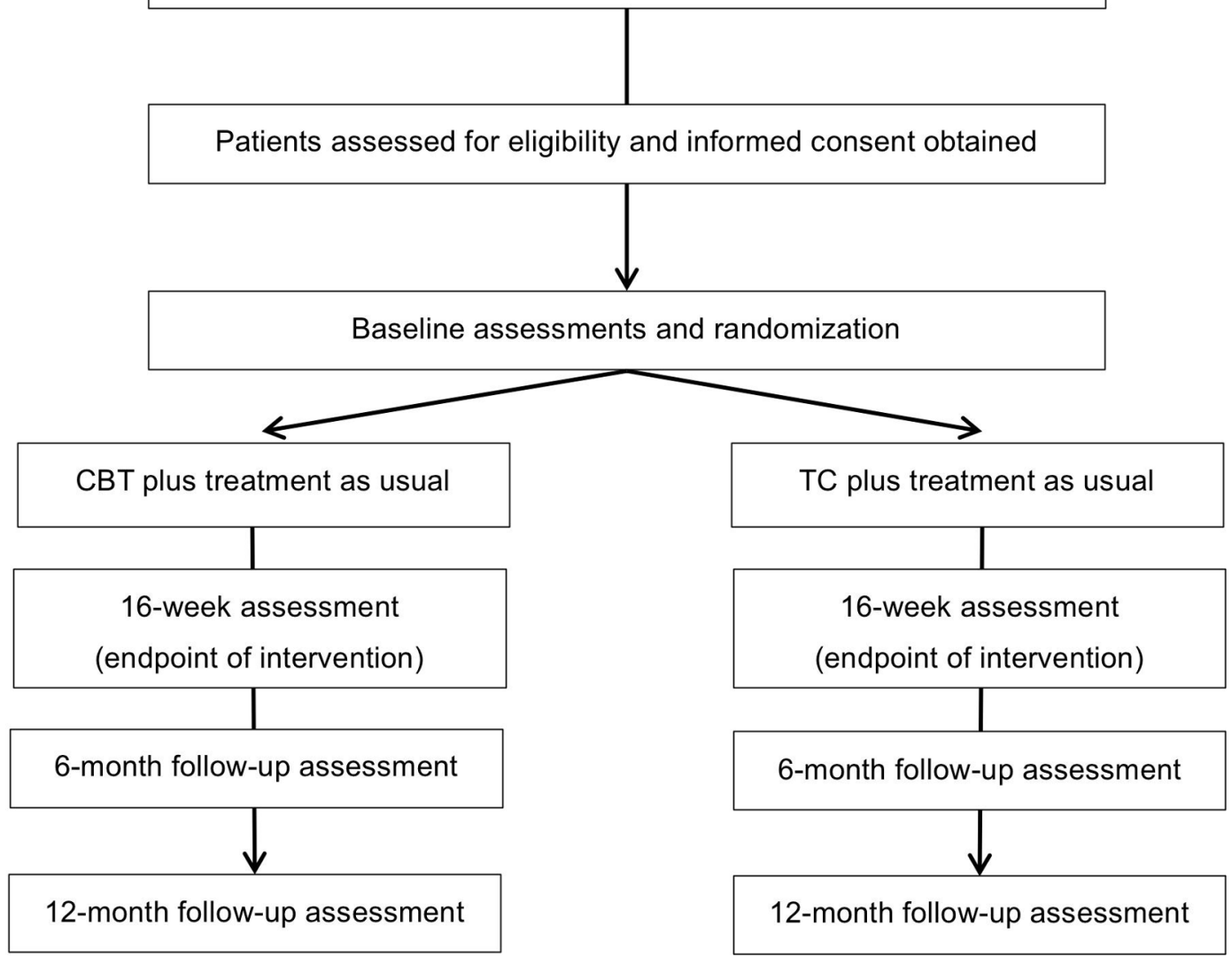

Figure 1 CONSORT diagram of patient flow through the study. CBT, cognitive behavioural therapy; CONSORT, Consolidated Standards of Reporting Trials; TC, talking control.

psychotic episodes; (2) have had a comorbid alcohol or substance use disorder in the 2 years prior to study entry; (3) have had any DSM-IV Axis I disorders other than MDD as the primary diagnosis, as assessed by the MiniInternational Neuropsychiatric Interview (MINI) ${ }^{4} 3031$ have antisocial personality disorder; (5) have serious and imminent suicidal ideation; (6) have a serious or unstable medical illness; (7) have had organic brain lesions or major cognitive deficits in the year prior to study entry; (8) have previously completed a full CBT programme; (9) are unlikely to attend more than eight visits during the 16-week trial phase (eg, due to relocation); (10) have MRI-specific contraindications, such as a pace maker or implanted metal. This study targeted patients with MDD in general clinical settings. Because there are limited number facilities that can provide CBT, pharmacotherapy is provided as first-line treatment; patients who do not sufficiently respond to pharmacotherapy alone must seek CBT in the clinical practices equipped to provide the treatment in Japan. In addition, the purpose of this study is to examine the effect of CBT and the difference between pharmacotherapy with CBT and pharmacotherapy with TC in typical medical settings. Therefore, we included patients on concurrent medications and those with any comorbid anxiety disorders, dysthymia, treatment-resistant depression or any personality disorders-except for antisocial personality disorder, which requires special management in general clinical settings.

\section{Procedures}

Recruitment

Treating psychiatrists will, during their usual consultations, provide a brochure with information about the study and invite patients to participate. If a patient shows interest in the study and provides contact details to the research team, a face-to-face appointment with a psychiatrist will be scheduled. After obtaining written informed consent, the patient will be assessed by the psychiatrist for eligibility. A diagnosis of MDD will be evaluated with the SCID Axis I Disorders ${ }^{27}$ while other Axis I disorders will be evaluated with MINI. ${ }^{30} 31$ Diagnostic interviews will be conducted by the study psychiatrists with extensive training in the administration of semistructured interviews.

\section{Randomisation}

All eligible patients will be randomly assigned to CBT with TAU or TC with TAU after the baseline assessment (1:1 allocation ratio). Block randomisation will be conducted by a computer-generated random number list using a 
freeware (http://www.randomization.com) prepared by a research assistant with no clinical involvement in the trial. Allocation will be stratified by the severity of the baseline GRID-HAMD ${ }_{17}$ score (severe vs non-severe). The cut-off GRID-HAMD ${ }_{17}$ score for the randomisation will remain undisclosed until the study termination to ensure concealment.

\section{Baseline assessment} Demographic data

The detailed demographic data will be collected as a part of the baseline assessment at study entry (see online supplementary file for details).

\section{Clinical and neurocognitive assessments}

Acute psychopathology will be assessed at study entry by the study psychiatrists or psychologists. Objective depressive symptoms will be assessed using the 17-item and 21-item GRID-HAMD. Patients' subjective perception of depression severity will be assessed using the self-reported Beck Depression Inventory-Second Edition (BDI-II) ${ }^{32} 33$ and 16-item Quick Inventory of Depressive Symptomatology Self-Reported (QIDS-SR ${ }_{16}$ ). ${ }^{34}{ }^{35}$ Health-related quality of life will be measured using the European Quality of Life Questionnaire-5 Dimensions (EQ-5D). ${ }^{36}$ The intensity of dysfunctional attitudes will be measured using the Dysfunctional Attitude Scale (DAS). ${ }^{38} 39$ Spontaneous negative self-statements and intrusive cognitions will be assessed using the Automatic Thoughts QuestionnaireRevised (ATQ-R). ${ }^{40}$ Neurocognitive functioning will be assessed using the Word Fluency Test (WFT), Digit Symbol Substitution Test (DSST) ${ }^{41}$ and Rey Auditory Verbal Learning Test (RAVLT). ${ }^{42}$ Assessments will also be conducted at 16 weeks after randomisation as well as at 6 and 12 months after the end of the 16-week intervention.

\section{Neuroimaging assessments}

Before conducting the functional MRI (fMRI) measurement, all paradigms will be explained to the patients. Images will be acquired on a 3.0 T GE Discovery MR750 MRI scanner with a 32-channel receiver coil in the MRI centre at the study hospital. A T2*-weighted echo planar imaging (EPI) sequence will be made to obtain the structural images necessary to locate functional activation. Functional-task MRI images of blood oxygenation leveldependent signals will be acquired using a T2*-weighted EPI sequence during a future-thinking task. Patients will also complete a $10 \mathrm{~min}$ eyes-open resting-state MRI scan. Detailed anatomical data will be collected using a highresolution T1-weighted image. The MRI session will last about $40 \mathrm{~min}$. MRI assessment will also be conducted 16 weeks after the randomisation (see online supplementary file for details).

\section{Future-thinking task}

Based on the Future Thinking Implicit Relations Assessment Procedure developed by Kosnes and colleagues, ${ }^{43}$ we will use a modified version of the future-thinking task contextualised and adapted to Japanese culture..$^{25}$ The future-thinking task uses an event design composed of four temporal conditions (distant future, near future, distant past and near past). Each run will last between 10.8 and $12.5 \mathrm{~min}$, depending on the time patients take to identify each event and to press a button in response (see online supplementary file for details).

\section{Treatment procedures}

Treatment will be conducted in an outpatient consultation room at the study hospital. During study treatment, other depression-specific empirical psychotherapy (ie, interpersonal therapy) and electroconvulsive therapy will be prohibited.

\section{Cognitive behavioural therapy}

Patients allocated to the CBT group will receive a course of 16 weekly $50 \mathrm{~min}$ face-to-face CBT sessions, with up to four additional sessions if deemed clinically appropriate by the study therapist (maximum of 20 sessions, minimum of 8 sessions). Therapists will follow the individual CBT treatment manual for major depression ${ }^{44}$; the manual was developed based on Beck's treatment manual ${ }^{45}$ with some adaptation to address the cultural characteristics of Japanese patients, such as a greater emphasis on interpersonal relationships and consideration of family as an essential part of treatment. ${ }^{46}$ Problem-solving techniques and specific approaches to address interpersonal issues and cognitive behavioural avoidance are emphasised. Therapists are encouraged to refer to the relevant approaches whenever necessary. Furthermore, the therapists are encouraged to give the patients feedback regarding the case conceptualisation and collaboratively set the treatment goal during the early phase of the programme.

\section{Talking control}

Patients allocated to the TC group will receive a course of 16 weekly 50-min face-to-face TC sessions, with up to 4 additional sessions if deemed clinically appropriate by the study therapist (maximum of 20 sessions, minimum of 8 sessions). Therapists will follow the individual TC intervention manual for depression developed by Serfaty et $a l .{ }^{47} \mathrm{TC}$ will be used as a comparison to evaluate the effectiveness of CBT. The manual specifies nine techniques to be used within a TC session (eg, sessions are client led, the therapist shows enthusiasm and interest towards the client, the therapist is non-judgmental) and 11 suggestions to avoid contaminating TC with other therapeutic techniques (eg, setting an agenda, applying specific cognitive psychotherapeutic and behavioural techniques, setting assignments). During TC sessions, clients will be encouraged to talk about any topic without interruption.

\section{Training and supervision of therapists}

Six therapists will deliver CBT and TC sessions: a psychiatrist $(n=1)$, a psychiatric nurse $(n=1)$ or master-level or doctoral-level clinical psychologists $(n=4)$. All therapists have received CBT training in the Keio University Cognitive Behavioral Therapy Training and Research Program and will receive supervision throughout the study. To 
ensure treatment fidelity, all therapists completed a 2-day workshop and will participate in 1-hour weekly group supervision sessions with other therapists during the study. During the group supervision sessions, therapists will present the case formulation and treatment plan. The group supervision sessions will be led by one of the authors (AN), the board member of trustees of the Japanese Association for Cognitive Therapy, who will encourage discussion of therapeutic difficulties and impasses, facilitate skill acquisition and provide peer support. To assess CBT competency, a random sample of audiotaped sessions will be rated using the Cognitive Therapy Rating Scale. ${ }^{45} 48$ All therapists have also participated in 3 hours of role play to master TC techniques. The therapists will be then supervised in 1-hour weekly group supervision sessions by NK, and audiotaped material from therapy sessions will be assessed using a fidelity checklist.

\section{Treatment as usual (usual depression care by psychiatrists)}

Although appropriate flexibility will be allowed for scheduling sessions, patients will typically receive biweekly, 10-15 min consultations with the treating psychiatrist during the treatment phase, with a minimum of eight consultations during the intervention phase. Although there will be no restriction of pharmacotherapy, it should be concordant with major practice guidelines for major depression, such as the American Psychiatric Association practice guideline ${ }^{5}$ (see online supplementary file for details).

\section{Follow-up phase treatment procedure}

There will be no restrictions on treatment options for the patients who receive care for their depression from the treating psychiatrists during this phase. Thus, the treating psychiatrists are allowed to refer the patients to appropriate mental health professionals for psychotherapy or electroconvulsive therapy if they deem it clinically appropriate. However, those who receive depression-specific empirical psychotherapy or electroconvulsive therapy will be recorded, and these will be considered deviations from the study protocol. However, the patient will not be considered to have dropped out of the study at this phase and will still receive protocol assessments.

\section{Discontinuations}

If a patient meets any one of the discontinuation criteria, the treating psychiatrist will discontinue the study intervention (see online supplementary file for details).

\section{Outcome measures}

The outcome measures are shown in table 1 .

\section{Primary outcome}

The primary outcome is the neural change in functional brain activity, especially in the areas associated with future thinking (eg, the BA10), as measured by fMRI data obtained from baseline (at randomisation) to 16 weeks postrandomisation (the end of the intervention).
Secondary outcomes

\section{Functional connectivity outcomes}

- Functional resting-state activity will be recorded. The change of functional connectivity in the brain areas related to future thinking from the baseline to 16 weeks postrandomisation will be assessed.

\section{Behavioral outcomes}

- All participant responses (yes or no) for each trial during the future-thinking task of the fMRI will be recorded. Change in the ratio of responses with negative valence to the total responses under each temporal condition from baseline to 16 weeks postrandomisation will be calculated.

- Reaction time (RT) will be calculated from the time at which the sentence is shown on the second slide to the time at which the participant pushes the button to respond. The change in RT to prompts of positive and negative valence under each temporal condition from baseline to 16 weeks postrandomisation will be evaluated.

\section{Clinical outcomes}

Patients will be assessed at four time-points: baseline (at randomisation); 16 weeks postrandomisation and 6 and 12 months after the end of 16-week intervention. All assessors will be blinded.

- The alleviation of depression symptoms as measured by changes in the total clinician-rated 17-item GRID-HAMD scores. All the assessors (psychiatrists and licensed clinical psychologists) have received extensive GRID-HAMD training and achieved excellent inter-rater reliability (Interclass correlation coefficients $($ ICC $)=0.94-0.98$ ). The GRID-HAMD will be conducted by central assessors by telephone. Patients will be instructed not to disclose their allocated treatment in the periodical assessments.

- The severity of subjective depression symptoms will be measured using the BDI-II and QIDS-SR ${ }_{16}$.

- The proportion of responders, defined as those with a $50 \%$ or greater reduction on the 17 -item and 21 -item GRID-HAMD, BDI-II and QIDS-SR ${ }_{16}$ relative to baseline will be analysed.

- The proportion of patients who achieve remission, defined as those with a 17-item GRID-HAMD score of $\leq 7,{ }^{49}$ BDI-II score of $\leq 13^{50}$ and QIDS-SR $_{16}$ of $\leq 5^{34}$ will be analysed.

- The degree of health-related quality of life will be measured using the EQ-5D.

- The pattern of automatic thought will be measured using the ATQ-R.

- The intensity of dysfunctional attitudes will be measured using the DAS.

\section{Neurocognitive evaluation}

- The level of neuropsychological function of the frontal cortex will be measured using the WFT and DSST. 
Table 1 Schedule of the assessments

\begin{tabular}{|c|c|c|c|c|c|c|}
\hline \multirow[b]{2}{*}{ Time-point } & \multirow{2}{*}{$\begin{array}{l}\text { Enrolment } \\
-1\end{array}$} & \multirow{2}{*}{$\begin{array}{l}\text { Baseline/ } \\
\text { randomisation } \\
0\end{array}$} & \multicolumn{2}{|l|}{ Intervention } & \multicolumn{2}{|l|}{ Follow-up } \\
\hline & & & 1-15weeks & 16 weeks & After 6 months & After 12 months \\
\hline \multicolumn{7}{|l|}{ Enrolment } \\
\hline Eligibility screen & $x$ & & & & & \\
\hline Informed consent & $x$ & & & & & \\
\hline Allocation & & $x$ & & & & \\
\hline CBT plus treatment as usual & & & 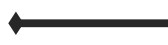 & $\checkmark$ & & \\
\hline TC plus treatment as usual & & & $\vdash$ & $\rightarrow$ & & \\
\hline \multicolumn{7}{|l|}{ Assessments } \\
\hline Demographic data & $x$ & & & & & \\
\hline Functional MRI & & $x$ & & $x$ & & \\
\hline \multicolumn{7}{|c|}{ Psychopathological assessments } \\
\hline GRID-HAMD & & $x$ & & $x$ & $x$ & $x$ \\
\hline BDI-II & & $x$ & & $\mathrm{x}$ & $\mathrm{x}$ & $x$ \\
\hline QIDS-SR16* & & $x$ & $x$ & $x$ & $x$ & $x$ \\
\hline$E Q-5 D$ & & $\mathrm{x}$ & & $\mathrm{x}$ & $x$ & $\mathrm{x}$ \\
\hline DAS & & $x$ & & $x$ & $x$ & $x$ \\
\hline ATQ-R & & $x$ & & $x$ & $x$ & $x$ \\
\hline WFT & & $x$ & & $x$ & $x$ & $x$ \\
\hline
\end{tabular}

${ }^{*}$ QIDS will also be assessed at each visit during the intervention phase.

ATQ-R, Automatic Thoughts Questionnaire-Revised; BDI-II, Beck Depression Inventory-Second Edition; CBT, Cognitive Behavioural Therapy; DAS, Dysfunctional Attitude Scale; DSM-IV, Diagnostic and Statistical Manual of Mental Disorders 4th Edition; DSST, Digit Symbol Substitution Test; EQ-5D, European Quality of Life Questionnaire-5 Dimensions; GRID-HAMD, GRID-Hamilton Depression Rating Scale; M.I.N.I, Mini-International Neuropsychiatric Interview; QIDS-SR16, 16-item Quick Inventory of Depressive Symptomatology Self-Reported; RAVLT, Rey Auditory Verbal Learning Test; SCID-I, Structured Clinical Interview for DSM-IV Axis I Disorders; TC, talking control; WFT, Word Fluency Test.

- The level and change in the degree of neuropsychological function of the lateral cortex will be measured using the RAVLT.

\section{Sample size estimation}

This trial is not confirmatory, and the sample size is mainly determined by taking account of feasibility. According to a review of sample size for $\mathrm{fMRI}$ studies, at least 12 subjects are required to achieve $80 \%$ power at the single-voxel level for typical activations to achieve a liberal threshold of 0.05 ; after correcting for multiple comparisons, the number of subjects should be larger to maintain this level of power. ${ }^{51} 52$ Therefore, we estimated our target number of patients will be 19 per group (a total of 38 patients) in which some exclusions up to $20 \%$ are allowed.

\section{Statistical analysis}

Task fMRI analysis

All preprocessing and analyses of imaging data will be conducted using the Statistical Parametric Mapping 12
(SPM12) implemented in MATLAB. Preprocessing will be conducted using the standard procedure, including slice-time correction, realignment, coregistration, spatial normalisation and smoothing. First-level analyses will be performed by an event-related model with four condition types: distant future, near future, distant past and near past. A general linear model will be used for statistical parametric maps. Subsequent second-level analyses will be performed on the SPM contrast images of the firstlevel canonical Hemodynamic Response Function (HRF) responses using paired t-tests. Group, time-point and group-by-time interaction will be included in the model as factors. The compound symmetry will be used as a covariance structure. Kenward-Roger adjustment for the denominator $\mathrm{df}$ will be used. $\mathrm{P}$ values of $<0.001$ will be considered statistically significant. Furthermore, region of interest analyses focused on the brain areas related to future thinking will be conducted to correct for multiple comparisons (see online supplementary file for details). 


\section{Resting fMRI analysis}

Resting-state fMRIs will be performed to explore functional connectivity in brain regions related to future thinking (see online supplementary file for details).

\section{Clinical and behavioural data analysis}

The analysis population in this study will be the intentionto-treat group, and all randomised patients will be included. For continuous outcomes, the least squares means and their 95\% CIs will be estimated using a mixedeffects model for repeated measures to detect changes from the baseline, which contains the treatment group, week and group-by-week interaction as fixed effects with an unstructured covariance matrix among the timepoints; Kenward-Roger df adjustment will be used. In addition, the model with stratified factors will be used for a sensitivity analysis. Mean changes for each group at each time-point and mean between-group differences will be estimated using appropriate contrasts in the MMRM which contains baseline value $(1 \mathrm{df})$, the treatment group, week and group-by-week interaction as fixed effects. Notably, missing values will not be inputted. For categorical outcomes, cross-tables between baseline and each time-point will be created for each group. Categorical outcomes will be analysed using the $\chi^{2}$ test or Fisher's exact test, and relative risks and their $95 \%$ CIs will be calculated. For binary outcomes, we will not use such likelihood-based model because the amount of information is limited with small sample binary data. Summary statistics (means and SD) of patient characteristics will be calculated. When appropriate, a t-test and Mann-Whitney U-test or analysis of covariance will be used to compare baseline continuous outcomes (means). The significance level will be set at 0.05 (two tailed). While the background factors might be controlled by randomisation, subgroup analyses will be conducted to determine whether any factors impacted the outcome. We will also perform perprotocol analyses in addition to the intention-to-treat analyses for primary outcomes to assess the influence of missing data on the results and other reasons accounting for non-conformance to the protocol.

Statistical analyses will be performed using SAS V.9.4 (SAS Institute).

\section{Data collection and management}

Please refer to the online supplementary file for information on data collection and management.

\section{ETHICS AND DISSEMINATION}

Before participation, all subjects will provide voluntary written informed consent after a discussion of the potential benefits and risks of participation (see online supplementary file for more details).

We will disseminate study findings to scientific and general audiences through major national and international medical conference presentations and by submission for publication in international peer-reviewed psychiatric and brain imaging journals using the International Committee of Medical Journal Editors author guidelines. Professional writers will not be used. The study will be reported according to the Consolidated Standards of Reporting Trials statement. We will also prepare lay summaries of study findings for dissemination to mental health consumer groups and for media release to the general public. We are not permitted to share study data with other individuals for any purpose without specific approval from the relevant data custodians and ethical review committee.

\section{Patient and public involvement}

Patients and the public were not involved in the development of the research questions, selection of outcome measures, study design, patient recruitment or conduction of the study. The burden of intervention was assessed by representatives of patient associations participating in the ethical review committee. As mentioned in the individual consent form, participants may obtain access to the final results of the study through the principal investigator.

\section{DISCUSSION}

The current study aims to provide a means by which to obtain new evidence of the neural and clinical effects of CBT for major depression in general psychiatric care settings. The study design is expected to detect meaningful differences in neural and clinical effectiveness outcomes. This study is distinguished from previous studies in that the design uses TC as a control group to evaluate the effectiveness of CBT, standardises psychiatric interviews to assess depression symptomatology, recruits patients with medium-to-severe depressive symptoms and evaluates the long-term effects of CBT for up to 12 months.

The study is subject to some challenges and limitations. The sample size is small due to the limited MRI machine time. We are aware that the single-site design compromises the generalisability of the results. We will not control for medication which may affect brain activity or connectivity. Although background factors are controlled by randomisation, the results of this study will be interpreted with caution. Nevertheless, this is the first design of a randomised controlled study to attempt to elucidate the neurobiological underpinnings of future-oriented pessimistic thinking using fMRI in patients with MDD following the receipt of CBT. The results of this study will improve the evidence-based knowledge of patients with depression.

\footnotetext{
Author affiliations

${ }^{1}$ Department of Neuropsychiatry, Keio University School of Medicine, Shinjuku-ku, Japan

${ }^{2}$ Clinical and Translational Research Center, Keio University Hospital, Shinjuku-ku, Japan

${ }^{3}$ Department of Psychology, Keio University Faculty of Letters, Minato-ku, Japan
} 
${ }^{4}$ Association of International Arts and Science, Yokohama City University School of Data Science, Yokohama, Japan

Acknowledgements The authors would like to thank our recruitment site for their support. We greatly appreciate the clinical evaluation staff at the Department of Neuropsychiatry, Keio University School of Medicine and National Center for Cognitive Behavior Therapy and Research, National Center of Neurology and Psychiatry. We are also thankful to the members of the Data Safety Monitoring Committee.

Contributors AN and NK conceived and designed the study. NK drafted the protocol manuscript and is organising the study. SU, YT, HT and TK refined the study protocol and study implementation. SU, YT and TA have provided methodological and statistical expertise. AN, NK and TA will conduct the statistical analyses. AN has provided CBT expertise and supervision of the therapists. AN has drafted the grant and is responsible for the implementation of the study. AN has been responsible for study management, staff training and supervision. NK, CK and YS have managed day-to-day study responsibilities, including monitoring recruitment, collecting data and liaising with recruitment sites. CK, DM, SN, SM, MO and YN will provide therapy. $\mathrm{DM}$ and $\mathrm{NI}$ will conduct data management and study monitoring. MM is the director of the site and provides clinical expertise and on-site management of the study. All authors critically reviewed and approved the final version of the manuscript.

Funding This research is supported by the Commissioned Research of National Institute of Information and Communications Technology grant: Research and development of technology for enhancing functional recovery of elderly and disabled people based on non-invasive brain imaging and robotic assistive devices. It is also supported by the Japan Society for the Promotion of Science KAKENHI grants (numbers 24120518, 24330210, 26780396 and JP17K04452), Inokashira Hospital Grants for Psychiatry Research and the Keio University School of Medicine Department of Neuropsychiatry Young Investigator Research Grant.

Disclaimer The funding sources no role in the study design, data collection, analysis and interpretation, writing of the report or the decision to submit the paper for publication.

Competing interests AN developed and wrote the Japanese CBT manual for depression and is involved in the National CBT Training and Supervision Project funded by the Japanese Ministry of Health Labour and Welfare.

Patient consent for publication Not required.

Ethics approval Approval of the study protocol was obtained from the Ethical Review Committee of Keio University School of Medicine (reference number 20150070).

Provenance and peer review Not commissioned; externally peer reviewed.

Open access This is an open access article distributed in accordance with the Creative Commons Attribution Non Commercial (CC BY-NC 4.0) license, which permits others to distribute, remix, adapt, build upon this work non-commercially, and license their derivative works on different terms, provided the original work is properly cited, appropriate credit is given, any changes made indicated, and the use is non-commercial. See: http://creativecommons.org/licenses/by-nc/4.0/.

ORCID iDs

Nariko Katayama http://orcid.org/0000-0003-2103-0287

Atsuo Nakagawa http://orcid.org/0000-0002-2294-2571

\section{REFERENCES}

1 World Health Organization. Depression: fact sheet. Geneva: World Health Organization, 2018. Available: http://www.who.int/ mediacentre/factsheets/fs369/en/ [Accessed 2 Feb 2019].

2 Gaynes BN, Warden D, Trivedi MH, et al. What did STAR*D teach us? Results from a large-scale, practical, clinical trial for patients with depression. Psychiatr Serv 2009;60:1439-45.

3 Beck AT. Cognitive therapy and the emotional disorders. New York: International Universities Press, 1976.

4 NICE. Depression: the treatment and management of depression in adults, clinical guideline 90. London: National Institute for Clinical Excellence, 2009.

5 APA. Practice guideline for the treatment of patients with major depressive disorder. 3rd edn. Washington, DC: American Psychiatric Association, 2010.

6 Cuijpers P, Berking M, Andersson G, et al. A meta-analysis of cognitive-behavioural therapy for adult depression, alone and in comparison with other treatments. Can J Psychiatry 2013;58:376-85.
7 Cuijpers P, Dekker J, Hollon SD, et al. Adding psychotherapy to pharmacotherapy in the treatment of depressive disorders in adults: a meta-analysis. J Clin Psychiatry 2009;70:1219-29.

8 Nakagawa A, Mitsuda D, Sado M, et al. Effectiveness of supplementary cognitive-behavioral therapy for pharmacotherapyresistant depression: a randomized controlled trial. J Clin Psychiatry 2017;78:1126-35.

9 Kupfer DJ, Frank E, Phillips ML. Major depressive disorder: new clinical, neurobiological, and treatment perspectives. Lancet 2012;379:1045-55.

10 DeRubeis RJ, Siegle GJ, Hollon SD. Cognitive therapy versus medication for depression: treatment outcomes and neural mechanisms. Nat Rev Neurosci 2008;9:788-96.

11 Dunlop BW, Rajendra JK, Craighead WE, et al. Functional connectivity of the subcallosal cingulate cortex and differential outcomes to treatment with cognitive-behavioral therapy or antidepressant medication for major depressive disorder. Am J Psychiatry 2017;174:533-45.

12 Kennedy SH, Konarski JZ, Segal ZV, et al. Differences in brain glucose metabolism between responders to CBT and venlafaxine in a 16-week randomized controlled trial. Am J Psychiatry 2007;164:778-88.

13 Chalah MA, Ayache SS. Disentangling the neural basis of cognitive behavioral therapy in psychiatric disorders: a focus on depression. Brain Sci 2018;8:150.

14 MacLeod AK, Tata P, Evans K, et al. Recovery of positive future thinking within a high-risk parasuicide group: results from a pilot randomized controlled trial. Br J Clin Psychol 1998;37:371-9.

15 Beck AT. Thinking and depression. I. idiosyncratic content and cognitive distortions. Arch Gen Psychiatry 1963;9:324-33.

16 Watkins E, Moberly NJ, Moulds ML. Processing mode causally influences emotional reactivity: distinct effects of abstract versus concrete construal on emotional response. Emotion 2008;8:364-78.

17 Watkins ER. Depressive rumination: investigating mechanisms to improve cognitive behavioural treatments. Cogn Behav Ther 2009;38:8-14.

18 Roepke AM, Seligman ME. Depression and prospection. Br J Clin Psychol 2016;55:23-48.

19 Altgassen M, Rendell PG, Bernhard A, et al. Future thinking improves prospective memory performance and plan enactment in older adults. Q J Exp Psychol 2015;68:192-204.

20 Addis DR, Wong AT, Schacter DL. Remembering the past and imagining the future: common and distinct neural substrates during event construction and elaboration. Neuropsychologia 2007;45:1363-77.

21 Gerlach KD, Spreng RN, Madore KP, et al. Future planning: default network activity couples with frontoparietal control network and reward-processing regions during process and outcome simulations. Soc Cogn Affect Neurosci 2014;9:1942-51.

22 Stawarczyk D, D'Argembeau A. Neural correlates of personal goal processing during episodic future thinking and mind-wandering: an ALE meta-analysis. Hum Brain Mapp 2015;36:2928-47.

23 Burgess PW, Dumontheil I, Gilbert SJ. The gateway hypothesis of rostral prefrontal cortex (area 10) function. Trends Cogn Sci 2007;11:290-8.

24 Gilbert SJ, Gonen-Yaacovi G, Benoit RG, et al. Distinct functional connectivity associated with lateral versus medial rostral prefrontal cortex: a meta-analysis. Neuroimage 2010;53:1359-67.

25 Katayama N, Nakagawa A, Umeda S, et al. Frontopolar cortex activation associated with pessimistic future-thinking in adults with major depressive disorder. Neuroimage Clin 2019;23:101877.

26 APA. Diagnostic and statistical manual of mental disorders. 4th edn Washington, DC: American Psychiatric Association, 1994.

27 First MB, Spitzer RL, Gibbon M, et al. Structured clinical interview for DSM-IV-TR axis I Disorders-Patient edition (SCID-I/P, 1/2007 revision). New York: Biometrics Research Department, 2007.

28 Tabuse $\mathrm{H}$, Kalali A, Azuma $\mathrm{H}$, et al. The new grid Hamilton rating scale for depression demonstrates excellent inter-rater reliability for inexperienced and experienced raters before and after training. Psychiatry Res 2007;153:61-7.

29 Williams JBW, Kobak KA, Bech P, et al. The GRID-HAMD: standardization of the Hamilton depression rating scale. Int Clin Psychopharmacol 2008;23:120-9.

30 Sheehan DV, Lecrubier Y, Harnett Sheehan K, et al. The validity of the mini international neuropsychiatric interview (MINI) according to the SCID-P and its reliability. European Psychiatry 1997;12:232-41.

31 Otsubo T, Tanaka K, Koda R, et al. Reliability and validity of Japanese version of the Mini-International neuropsychiatric interview. Psychiatry Clin Neurosci 2005;59:517-26.

32 Beck AT, Steer RA, Brown GK. Manual for the Beck depression Inventory-II (BDI-II). San Antonio, TX: Psychological Corporation, 1996. 
33 Kojima M, Furukawa TA, Takahashi $\mathrm{H}$, et al. Cross-cultural validation of the Beck depression Inventory-II in Japan. Psychiatry Res 2002;110:291-9.

34 Rush AJ, Trivedi MH, Ibrahim HM, et al. The 16-Item quick inventory of depressive symptomatology (QIDS), clinician rating (QIDS-C), and self-report (QIDS-SR): a psychometric evaluation in patients with chronic major depression. Biol Psychiatry 2003;54:573-83.

35 Fujisawa D, Nakagawa A, Tajima M, et al. Development of the Japanese version of quick inventory of depressive symptomatology self-reported (QIDS-SR16-J) (in Japanese). Stress Science 2010;25:43-52

36 EuroQol Group. EuroQol - a new facility for the measurement of health-related quality of life. Health Policy 1990;16:199-208.

37 Fukuhara S, Ikegami N, Torrance GW, et al. The development and use of quality-of-life measures to evaluate health outcomes in Japan. Pharmacoeconomics 2002;20:17-23.

38 Tajima M, Akiyama T, Numa $\mathrm{H}$, et al. Reliability and validity of the Japanese version of the 24-item dysfunctional attitude scale. Acta Neuropsychiatr 2007;19:362-7.

39 Weissman A. The dysfunctional attitude scale: a validation study. Dissertation Abstracts International 1979;40:1389-90.

40 Harrell TH, Ryon NB. Cognitive-behavioral assessment of depression: clinical validation of the automatic thoughts questionnaire. J Consult Clin Psychol 1983;51:721-5.

41 McLeod DR, Griffiths RR, Bigelow GE, et al. An automated version of the digit symbol substitution test (DSST). Behav Res Methods Instrum 1982;14:463-6.

42 Schmidt M. Rey auditory verbal learning test: a Handbook. Los Angeles, CA: Western Psychological Services, 1996.
43 Kosnes L, Whelan R, O'Donovan A, et al. Implicit measurement of positive and negative future thinking as a predictor of depressive symptoms and hopelessness. Conscious Cogn 2013;22:898-912.

44 Nakagawa A, Sado M, Mitsuda D, et al. Effectiveness of cognitive behavioural therapy augmentation in major depression treatment (ECAM study): study protocol for a randomised clinical trial. BMJ Open 2014:4:e06359.

45 Beck AT, Rush AJ, Shaw BF, et al. Cognitive therapy of depression. New York: Guilford Press, 1979.

46 Fujisawa D, Nakagawa A, Tajima M, et al. Cognitive behavioral therapy for depression among adults in Japanese clinical settings: a single-group study. BMC Res Notes 2010;3:160.

47 Serfaty M, Csipke E, Haworth D, et al. A talking control for use in evaluating the effectiveness of cognitive-behavioral therapy. Behav Res Ther 2011:49:433-40.

48 Young J, Beck AT. Cognitive therapy rating scale manual. Philadelphia: University of Pennsylvania, Psychotherapy Rsearch Unit, 1980.

49 Frank E, Prien RF, Jarrett RB, et al. Conceptualization and rationale for consensus definitions of terms in major depressive disorder. Remission, recovery, relapse, and recurrence. Arch Gen Psychiatry 1991;48:851-5.

50 Hiroe T, Kojima M, Yamamoto I, et al. Gradations of clinical severity and sensitivity to change assessed with the Beck depression Inventory-II in Japanese patients with depression. Psychiatry Res 2005; 135:229-35.

51 Desmond JE, Glover GH. Estimating sample size in functional MRI (fMRI) neuroimaging studies: statistical power analyses. J Neurosci Methods 2002;118:115-28.

52 Friston KJ, Holmes AP, Worsley KJ. How many subjects constitute a study? Neuroimage 1999;10:1-5. 